\title{
Digestión secundaria en el análisis del contenido gástrico del calamar rojo Dosidicus gigas (Cephalopoda: Teuthoidea): ¿qué tan importante es?
}

Secondary digestion in the analysis of the stomach contents of jumbo flying squid Dosidicus gigas (Cephalopoda: Teuthoidea): how important is it?

\section{Patricio Ulloa, ${ }^{1,2}$, Víctor H. Ruiz ${ }^{3}$ y Ricardo Figueroa ${ }^{4}$}

\author{
${ }^{1}$ Programa Doctorado en Sistemática y Biodiversidad, Escuela de Graduados, Departamento de Zoología, Universidad de \\ Concepción, Casilla 160-C, Concepción, Chile. pulloa@udec.cl \\ ${ }^{2}$ Servicio Nacional de Pesca, Dirección Regional VIII, Casilla 52, Talcahuano, Chile. pulloa@sernapesca.cl \\ ${ }^{3}$ Departamento de Zoología, Universidad de Concepción, Casilla 160-C, Concepción, Chile \\ ${ }^{4}$ Unidad de Sistemas Acuáticos, Centro EULA-Chile, Universidad de Concepción, Casilla 160-C, Concepción, Chile
}

\begin{abstract}
The biological significance of the secondary digestion, i.e., prey of the prey, was determined in the analysis of stomach contents of 112 jumbo squid Dosidicus gigas. Squids were caught on the continental shelf off the coast of centralsouthern Chile during austral winter and spring 2005. All prey (digested and those from opened stomachs of the prey in the squids) found in the squid stomachs were assessed. The results obtained by using several trophic indices (numeric, gravimetric, frequency of occurrence, relative importance, percentage similarity and trophic heterogeneity), plus the average weight of common prey between seasons (winter and spring) and the Spearman correlation coefficient, revealed no significant differences in the feeding behavior between sexes, ontogenetic and seasonal groups. Comparing the primary with the secondary digestion allow us to conclude that consider or not the latter, in a quantitative point of view, is not relevant in assessing the food preferences squid. On the other hand, the biological significance of secondary digestion from a qualitative point of view may still be relevant when the aim is to avoid overestimating the food spectrum for this species.
\end{abstract}

Key words: Stomach contents, preys, diet, season

\begin{abstract}
Resumen.- Se determinó la importancia biológica de la digestión secundaria, i.e., presas de las presas, en el análisis de los contenidos estomacales de 112 individuos del calamar rojo Dosidicus gigas, capturados sobre la plataforma continental, frente a la costa Centro-Sur de Chile, durante invierno y primavera austral de 2005. Todas las presas (digeridas y aquellas de los estómagos abiertos de las presas del calamar) halladas en los contenidos estomacales de los calamares fueron analizadas. Los resultados obtenidos mediante el uso de varios índices tróficos (numérico, gravimétrico, frecuencia de ocurrencia, importancia relativa, similitud porcentual, heterogeneidad trófica), el peso medio de las presas comunes entre estaciones (invierno y primavera) y el coeficiente de correlación de Spearman entre presas, no mostraron diferencias significativas en el comportamiento alimentario del calamar rojo, respecto de su condición sexual, ontogenética y estacional. Comparar la digestión primaria con la secundaria permite concluir que considerar o no a esta última, desde un punto de vista cuantitativo, no es relevante en la evaluación de las preferencias alimentarias del calamar. Por otro lado, la importancia biológica de la digestión secundaria desde un punto de vista cualitativo, podría ser relevante en el caso de evitar la sobreestimación del espectro alimentario de esta especie.
\end{abstract}

Palabras clave: Contenido estomacal, presas, dieta, estación

\section{INTRODUCCIÓN}

Los estudios de contenidos estomacales constituyen uno de los aspectos biológicos más relevantes dentro de la ecología y dinámica trófica (Crespin de Billy et al. 2000). Establecer las relaciones de tipo alimentarias de una determinada especie al interior de su comunidad, en función de la explotación de las fuentes disponibles, permite evaluar el nicho ecológico que ésta ocupa (Finke
\& Snyder 2008). A nivel poblacional, los requerimientos de recursos alimentarios son parte relevante para el entendimiento del concepto de nicho. En particular, cuando éste se asocia con una tasa de crecimiento positiva, y el impacto asociado al mismo involucra una conceptualización de las condiciones ambientales que en definitiva permitirán la sobrevivencia de la población, 
donde se incluyen las relaciones tróficas (Soberon \& Nakamura 2009). Adicionalmente, tanto en los modelos biológico-pesqueros tradicionales, así como en aquellos que incorporan parámetros ambientales en la evaluación de los stocks, reconocen dentro del ambiente biológico la importancia de las relaciones depredador-presa (Lotka 1925, Volterra 1926, Wiff \& Quiñones 2004). En estas relaciones, el estudio de los contenidos estomacales forma parte relevante en su comprensión (Pardo-Gandarillas et al. 2007a), siendo utilizados también para comprender los ciclos en la transmisión de los parásitos en los distintos hospedadores, posibilitando con ésto la caracterización del nicho trófico de los huéspedes (Pardo-Gandarillas et al. 2004, 2009), el establecimiento del orden del consumidor en su nicho trófico (Nigmatullin et al. 2009) y en la obtención de información biológica sobre las presas que consumen (Polo-Silva et al. 2007). De acuerdo a Labbé \& Arana (2001), estos estudios no sólo permiten establecer las preferencias alimentarias de los recursos marinos, sino también conocer las especies que habitan estos lugares y que muchas veces no son extraídos por las artes o sistemas de pesca utilizados.

En general, los estudios de contenidos estomacales, suponen que todas las presas contenidas en los tractos digestivos de una determinada especie han sido capturadas y consumidas por dicho depredador (Clarke et al. 1976, 1988, Clarke \& Paliza 2000). Así, la digestión secundaria o hipótesis de 'la presa de la presa' que dice relación con aquellos contenidos estomacales del tracto digestivo de un depredador, que fueron consumidos por una de sus presas y no en forma directa por éste (Smith \& Whitehead 2000), prácticamente no ha sido considerada en los estudios de carácter trófico (Clarke et al. 1988, Clarke \& Paliza 2000, Smith \& Whitehead 2000, Santos et al. 2001). Esto puede observarse, a modo de ejemplo, en algunos trabajos clásicos sobre la alimentación de cachalotes, principal depredador de los calamares (Clarke et al. 1976, 1988, Clarke 1997, Clarke \& Pascoe 1997, Clarke \& Paliza 2000) en donde Dosidicus gigas es su principal presa (Clarke et al. 1988, Ruiz-Cooley et al. 2004). De esta forma, la discusión sobre si algunas presas debieran o no ser consideradas como provenientes de una ingestión secundaria, permanece incierto (Clarke et al. 1988, 1993, Clarke \& Paliza 2000).

El calamar rojo Dosidicus gigas (D’Orbigny, 1835) es un depredador de alto nivel trófico en las aguas del Pacífico Oriental tiene un rol preponderante en la estructura trófica de los ecosistemas marinos para dicha zona (Roper et al. 1984, Fernández \& Vásquez 1995, Nigmatullin et al. 2001, Bazzino et al. 2007, Ibañez et al.
2008), por lo tanto, constituye un modelo interesante para el análisis de la digestión secundaria, ya que permitiría probar la importancia biológica que esta digestión reviste en los estudios de contenidos estomacales y contribuiría en la descripción precisa del espectro trófico de la especie. De este modo, el objetivo de este estudio fue describir el espectro trófico del calamar rojo considerando la relevancia cuantitativa y cualitativa de la digestión secundaria, además de la importancia de factores como el sexo y la estacionalidad en dicha descripción. En este último punto, la segregación por sexo, tamaño corporal y estacionalidad en el muestreo, fue considerada en atención a que en varios trabajos sobre alimentación de $D$. gigas, resultaron relevantes al momento de establecer conclusiones sobre el comportamiento alimentario del calamar, ya sea en relación a su estructura poblacional, madurez sexual, biología reproductiva, migraciones estacionales de tipo alimentaria y/o de desove (Wormuth 1976, Ehrhardt 1991, Clarke \& Paliza 2000, Nigmatullin et al. 2001, Shukhgalter \& Nigmatullin 2001, Taipe et al. 2001, Markaida \& Sosa-Nishizaki 2003, González \& Chong 2006, Markaida 2006, Bazzino et al. 2007).

\section{Materiales y Métodos}

Se recolectó un total de 112 calamares rojos, desde lances de pesca efectuados por naves industriales merluceras que operan con redes de pesca de arrastre de fondo tipo 'arrow', en el área de la plataforma continental de la zona centro sur de Chile $\left(36^{\circ}-39^{\circ} \mathrm{S}\right)$, a una profundidad media de $230 \mathrm{~m}$, durante las estaciones de invierno (mes de julio) y primavera (mes de octubre) del 2005 y en donde habitualmente aparece el calamar rojo entre las capturas de la merluza común, Merluccius gayi (Guichenot, 1848), como especie objetivo. Se efectuaron muestreos en 2 áreas de pesca en invierno ( $38^{\circ} 55^{\prime} \mathrm{S}$; $73^{\circ} 56^{\prime} \mathrm{O}$ y $36^{\circ} 47^{\prime} \mathrm{S}$; $73^{\circ} 43^{\prime} \mathrm{O}$ ) y 3 áreas de pesca en primavera (36 $46^{\circ} \mathrm{S}$; $73^{\circ} 44^{\prime} \mathrm{O}$,

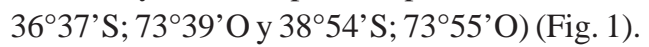

Para la elección del tamaño de muestra, se consideró el método gráfico de Hoffman (1979), el cual sugiere un mínimo de 20 a 25 estómagos por mes muestreado, para su conservación en formaldehído y de 20 estómagos para su conservación bajo congelamiento. Este método ha sido usado tanto para el análisis de contenidos estomacales en $D$. gigas, así como en estudios de su fauna parasitaria (Markaida 2001, Shukhgalter \& Nigmatullin 2001, Markaida \& Sosa-Nishizaki 2003, Cubillos et al. 2004). Además, se analizó la riqueza bruta acumulada de ítemes presa, en función del incremento del tamaño de la muestra (Ulloa et al. 2006). 


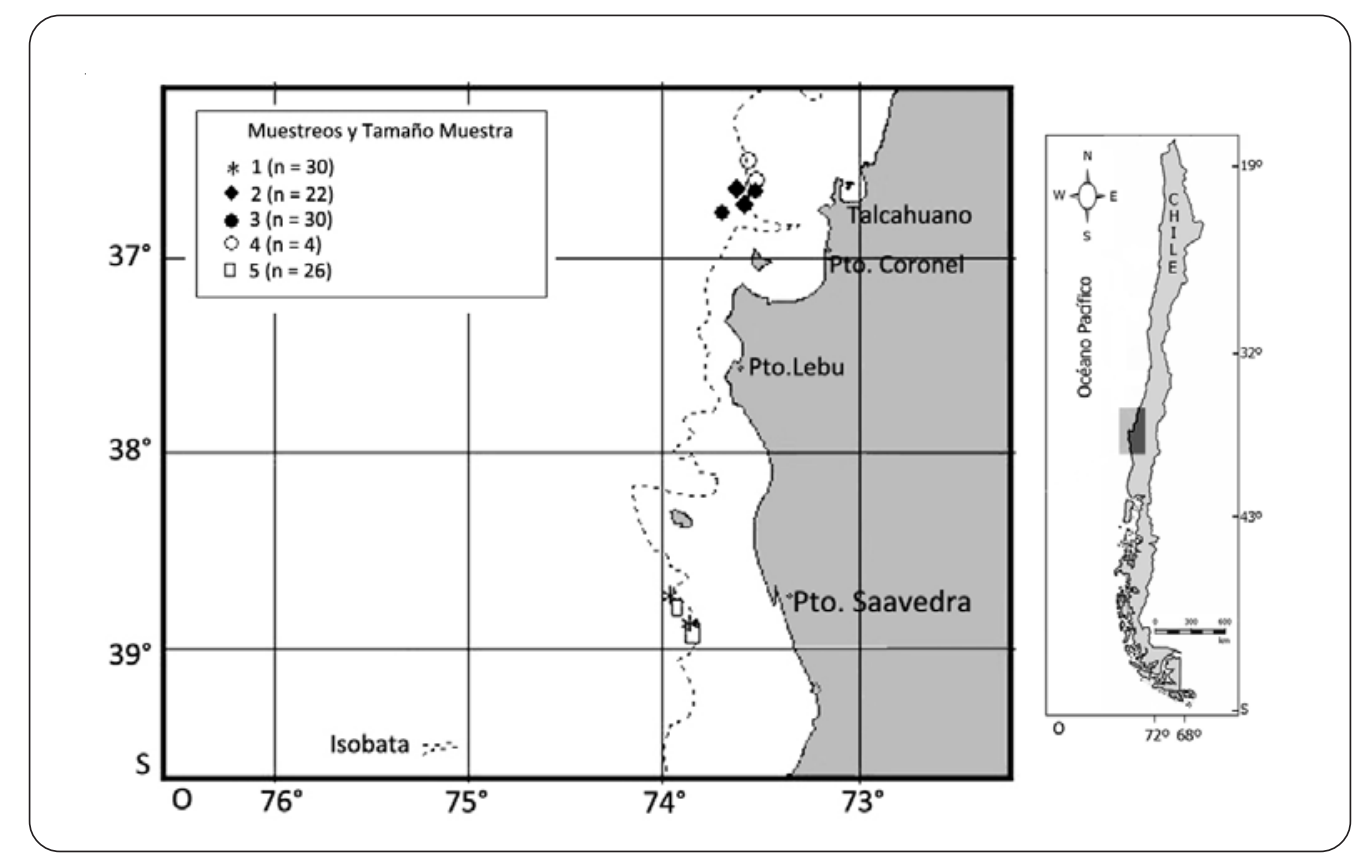

Figura 1. Mapa con los sitios de muestreo de Dosidicus gigas en las estaciones de invierno (1 y 2) y de primavera $(3,4$ y 5) durante 2005. Cada símbolo señala donde se realizaron los muestreos / Map showing the sampled locations of Dosidicus gigas captured in winter (1 and 2) and spring (3,4 and 5), during 2005. Each symbol indicates where the trawlings were perfomed

Los individuos recolectados fueron medidos en longitud de manto dorsal (LM), pesados y sexados, para luego extraer sus estómagos, los que fueron mantenidos en formol diluido al 10\%. Los estómagos de los calamares fueron abiertos por su lado ventral. Los contenidos estomacales fueron vaciados en un cedazo de $0,5 \mathrm{~mm}$ de abertura de malla y vistos en lupa estereoscópica Zeiss ${ }^{\circledR}$ con un aumento de 40X y a ojo desnudo. Para identificar las presas, se consideró el criterio del nivel taxonómico más bajo posible, con literatura especializada, según los grupos siguientes: i) peces (Wisner 1971, 1974, Rojo 1976, Sáez 1982, Leible \& Miranda 1989, Cohen et al. 1990, Nelson 1994, Falabella et al. 1995, Ruiz et al. 1995); ii) moluscos (Roper et al. 1969, 1984, Wolff 1982, Brunetti et al. 1999, Markaida 2001) y, iii) crustáceos (Bahamonde 1968, Retamal 1977, 1981, Méndez 1981, Bowman \& Abele 1982). Además, se usaron muestras referenciales de los propios autores, preparaciones del Museo de Zoología de la Universidad de Concepción (MZUC-UCCC) y la colaboración de especialistas de esta Universidad, así como externos.

Las presas presentes en el contenido estomacal fueron contadas y pesadas (peso húmedo) con una balanza de 0,0001 g de sensibilidad. Para evitar imprecisiones, en particular en aquellos casos con alto grado de digestión, se utilizó como criterio, la identificación de estructuras permanentes y siempre presentes en estos contenidos estomacales, tales como ojos, exoesqueletos, huesos, otolitos, espinas, restos de piel, intestinos y coloraciones de la masa carnosa, en relación con muestras patrones y con otros contenidos estomacales con menor grado de digestión, teniendo en consideración en el caso de los otolitos, las limitantes vinculadas a su uso (Jobling \& Breiby 1986, Granadeiro \& Silva 2000).

Con el objeto de determinar las preferencias alimentarias de Dosidicus gigas, se utilizaron los siguientes descriptores tróficos: i) método numérico $\mathrm{N}$ (número de individuos de un ítem presa presentes expresado como porcentaje del total de estómagos con contenido); ii) método gravimétrico G (peso total de un ítem presa expresado como porcentaje respecto al peso total de todos los ítemes presa encontrados en los estómagos con contenido); iii) método de frecuencia de ocurrencia $F$ (número de estómagos en que un determinado ítem presa aparece con respecto al total de estómagos con contenido, expresado como porcentaje); iv) índice de importancia relativa IIR ((\%N+\%G)*\%F); v) similitud porcentual ISP (sumatoria de los IIR más bajo de 
cada una de las presas que fueron compartidas entre dos grupos de peces; el criterio de una alta similitud fue dado para valores mayores al 50\%), y, vi) índice de diversidad de Shannon-Wiener H (expresa la riqueza de los ítemes presa y la cantidad o abundancia relativa de individuos para cada ítem presa expresada en peso) (Whittaker 1952 fide González \& Chong 1997, Berg 1979, Hyslop 1980, Bloom 1981, Krebs 1985, Zar 1999, Moreno 2001).

Para determinar la existencia de digestión secundaria ('presas secundarias' que han quedado entre los contenidos estomacales, una vez que se han digerido las presas 'primarias'), se utilizó como método de reconocimiento los restos alimentarios presentes en los tractos digestivos abiertos de las presas contenidas en los estómagos de los calamares, de esta forma su presencia entre las presas propias del individuo de calamar muestreado podría ser erróneamente considerada como parte de la digestión primaria. Aquellos estómagos de las presas que se encontraban cerrados no fueron considerados en el análisis, salvo para fines comparativos respecto del tipo y estado de la presa en aquellos estómagos que se encontraron abiertos. Posteriormente, y para establecer la importancia de este tipo de digestión, se llevó a cabo un análisis comparativo con relación a las variables sexo, tamaño y estacionalidad (invierno o primavera), en donde en un escenario se asumió sólo la existencia de la digestión primaria y en otro, se consideró además la existencia de las 'presas de las presas' o 'digestión secundaria'. En el primer caso, todas las presas fueron consideradas como capturadas por cada individuo de calamar rojo y en el segundo, fueron eliminadas todas las presas en las cuales se determinó que su origen fue el contenido estomacal de alguna de las presas presentes en los estómagos del individuo de calamar rojo y no debido a la captura directa por parte del calamar.

Se utilizaron los índices ISP, H y F, para establecer el grado de similitud (\%), heterogeneidad y la frecuencia trófica entre machos y hembras, tanto en invierno como en primavera. Los valores $\mathrm{H}$, fueron comparados a través del cálculo de la diferencia entre las varianzas del $\mathrm{H}$ ponderado, siendo evaluada tal diferencia mediante la prueba de t-Student y se utilizaron además con fines comparativos, el Hmax y la equidad E. El descriptor trófico F, fue comparado entre sexos, mediante la prueba de $\chi^{2}$ (Cid et al. 1990) y utilizando como variable la frecuencia de aparición de todas las presas. Dado que no se encontraron diferencias significativas en la frecuencia de ocurrencia de las presas en las distintas áreas de pesca, tanto en invierno como en primavera $\left(\chi^{2}=18,18\right.$; g.l. $=9$; $P$ $>0,05$ ), no se consideraron en el análisis los 5 sitios de muestreo en forma individual, sino sólo su agrupamiento a nivel de la estacionalidad ya definida. La conformación de grupos de tamaños corporales (medido como longitud del manto), fueron determinadas como discretas Unidades Ontogenéticas Tróficas (OTUs). Dentro de la estación de invierno y primavera, se consideraron como criterios de agrupamiento, intentar obtener un tamaño de muestra adecuado para cada una de las clases de tamaño previamente establecidas, privilegiar el grado de similitud alimentaria para su conformación (mediante el uso del ISP) y considerar aquellos antecedentes bibliográficos que apoyaran la consideración de tales clases basado principalmente en las afinidades de tipo reproductivas y alimentarias de la especies en función de cada rango de LM. Así por ejemplo, en la estación de invierno, las clases de LM fueron agrupadas ya sea del punto de vista de su ubicación en el limite inferior de la estructura intraespecífica de los individuos adultos de la especie (Argüelles et al. 2001, Nigmatullin et al. 2001), considerando además aspectos de tipo reproductivos y/o de estructura de cohortes (Bojórquez et al. 2001, González \& Chong 2006, Bazzino et al. 2007). Consecuentemente, la estructuración de unidades discretas para la estación de invierno incluyó 6 clases (clase 1: $\leq 65 \mathrm{~cm} \mathrm{LM}$; clase 2: 66-70 cm LM; clase 3: 71-75 cm LM; clase 4: 76-80 cm LM; clase 5: 81-85 cm LM y clase 6: 86-90 cm LM) y la primavera 5 (clase 1: $\leq 61 \mathrm{~cm}$ LM; clase 2: 62-70 cm LM; clase 3: 7176 cm LM; clase 4: 77-82 cm LM y clase 5: 83-94 cm LM).

Para el análisis de la variación trófica en Dosidicus gigas en invierno y primavera, se consideró la significancia en la dieta del calamar rojo, de las variables sexo y condición ontogenética. El análisis incluyó el estudio del descriptor trófico F, así como el análisis del ISP entre las clases de tamaño en invierno y en primavera y entre estas estaciones del año. Adicionalmente, se efectuaron análisis comparativos de los pesos medios de los ítemes presas comunes tomados en muestras independientes, mediante el test no paramétrico de MannWhitney y del grado de asociación trófica de tales presas, mediante el cálculo del coeficiente de correlación lineal de rangos de Spearman (Zar 1999), usando como variable al IIR (\%). Finalmente, se realizó un análisis comparativo del espectro trófico del calamar considerando e ignorando la digestión secundaria, a través del análisis de la presencia-ausencia de presas. 


\section{Resultados}

La riqueza bruta de presas acumulada mostró estabilidad en el invierno cuando se muestreó el estómago número 50 (de un total de 52) y en la primavera el número 52 (de un total de 60). La LM del calamar rojo, variaron entre 52 y $94 \mathrm{~cm}$, con un promedio de 74,5 cm (D.E. = 7,78; $\mathrm{n}=112$ ). La LM promedio de machos y hembras (Fig. 2) no fue significativamente diferente entre estaciones (U de MannWhitney = 1735; $P>0,05$ ). El análisis de los 112 estómagos (todos con contenido estomacal) mostró la existencia de 30 ítemes presas (descontando las presas de la digestión secundaria), distribuidos en las estaciones de invierno y primavera (Tabla 1).

El grupo principal de presas lo constituyeron los peces ( $\mathrm{N}=88,36 \%, \mathrm{~F}=97,32 \%$ ), seguido secundariamente de los crustáceos ( $\mathrm{N}=8,58 \%, \mathrm{~F}=20,53 \%)$ y de los moluscos $(\mathrm{N}=3,05 \%, \mathrm{~F}=36,61 \%)$ (Tabla 1). En primavera, las presas de segundo orden como los crustáceos tuvieron una mayor relevancia a través de los descriptores utilizados, observándose además una mayor riqueza de ítemes presa en dicha estación (Tabla 1). Las presas secundarias presentes en los estómagos de Dosidicus gigas estuvo compuesta principalmente por peces, con una mayor riqueza de presas en primavera (Tabla 2).

De los 30 ítemes presas de tipo primario detectados en los estómagos de Dosidicus gigas (Tabla 1), 12 fueron hallados como presas secundarias (Tabla 2): Teleostei sin identificar (s/i), Stomiiformes, Clupeidae, Gonostomatidae, Myctophidae (s/i), Stomias sp., Hygophum sp., M. gayi, Caridea, Gammaridae, Benthesicymidae y Oplophorus novaezeelandiae. Asimismo, un total de 8 ítemes presa fueron encontrados exclusivamente como presas secundarias (Tabla 2): Diaphus sp., Lampadena sp., Binghamichthys sp., Crustacea, Euphausiacea, Anomura, Pterygosquilla armata H. Milne Edwards, 1837 y Pleuroncodes monodon H. Milne Edwards, 1837. El número de estómagos en donde se detectó la existencia de la ingestión secundaria alcanzó un 33,93\% ( $\mathrm{n}=38$ estómagos).

\section{ANÁLISIS DEL ESPECTRo TRÓfico gENERAL DE $\boldsymbol{D}$. gigas}

En el caso de considerar a todas las presas de la digestión primaria, el número de ítemes presa fue de 38 (Tablas 1 y 2). En la estación de invierno, el número de ítemes presa alcanzó los 17 y en primavera 32. Al considerar la digestión secundaria, el número total de ítemes presa se redujo a un total de 30 .

En la estación de invierno se reveló la existencia de 10 ítemes presa: Teleostei (s/i), Myctophidae (s/i), Diaphus sp., Hygophum sp., Lampadena sp., M. gayi (Guichenot, 1848), Crustacea (s/i), Amphipoda: Gammaridae, P. armata y P. monodon) y, 14 en la primavera (Stomiiformes (s/i), Myctophidae, Gonostomatidae, Clupeidae, Stomias sp., Binghamichthys sp., Diaphus sp., M. gayi, Euphausiacea, Caridea, Anomura, Benthesicymidae, Oplophorus novaezeelandiae De Mann, 1931, y P. monodon. En términos generales cuando se comparó la dieta según los tipos de digestión (sólo primaria vs primaria y secundaria), hubo una alta similitud dietaria (ISP) entre sexos: 89,1\% sólo digestión primaria vs 90,6\% con ambos tipos de digestión. En el caso de la LM, las clases mas pequeñas (1 y 2) mostraron baja similitud en relación a las otras clases de LM, con valores similares en ambos tipos de digestión (19,23\% vs 82,13\% sólo digestión primaria y
Figura 2. Distribución de frecuencia de la longitud del manto de 112 especímenes de Dosidicus gigas / Frequency distribution of the mantle length of 112 specimens of jumbo squid Dosidicus gigas

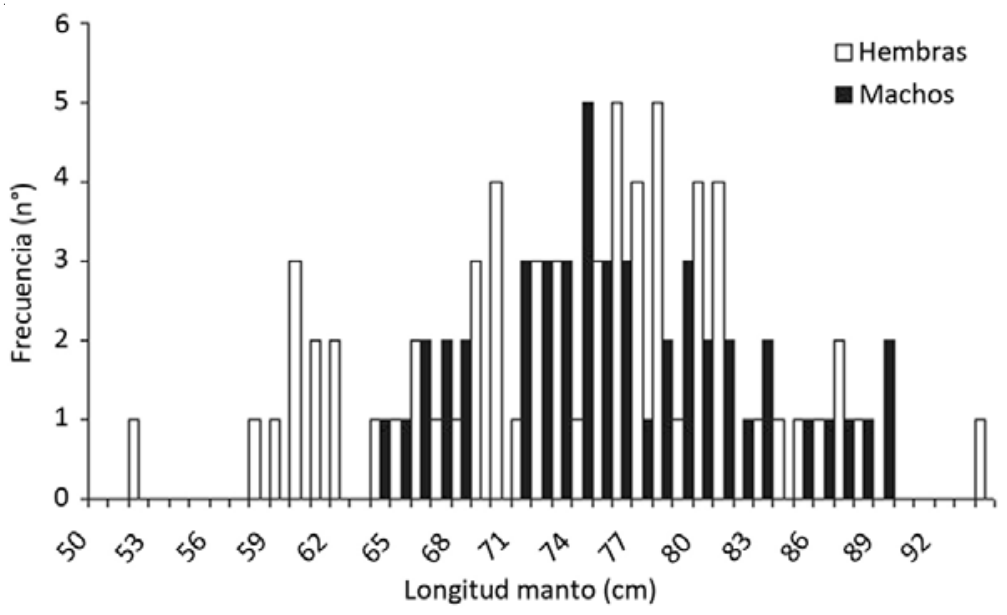

Vol. 47, №3, 2012

Revista de Biología Marina y Oceanografía 
Tabla 1. Ítemes presa $(n=30)$ presentes en los 112 estómagos de Dosidicus gigas muestreados durante las estaciones de invierno y primavera de 2005 / Prey items $(n=30)$ found in the 112 jumbo squid stomachs sampled during the winter and spring season 2005

\begin{tabular}{|c|c|c|c|c|}
\hline \multirow[t]{2}{*}{ Ítem Presa } & \multicolumn{2}{|c|}{ Invierno (*) } & \multicolumn{2}{|c|}{ Primavera $(* *)$} \\
\hline & $\mathrm{N}(\%)$ & $\mathrm{F}(\%)$ & $\mathrm{N}(\%)$ & $\mathrm{F}(\%)$ \\
\hline Pisces & 91,14 & 96,15 & 88 & 98,33 \\
\hline Infraclase Teleostei (s/i) & 9,52 & 17,31 & 0,08 & 1,67 \\
\hline Orden Gadiformes & & & 0,24 & 5,00 \\
\hline Orden Perciformes & & & 0,16 & 1,67 \\
\hline Orden Stomiiformes & & & 0,08 & 1,67 \\
\hline Familia Stomiidae (s/i) & & & 15,15 & 3,33 \\
\hline Stomias sp. 1 & & & 56,76 & 36,67 \\
\hline Stomias sp.2 & & & 0,24 & 3,33 \\
\hline Stomias sp.3 & & & 0,16 & 1,67 \\
\hline Familia Clupeidae & & & 0,08 & 1,67 \\
\hline Familia Gonostomatidae & & & 0,49 & 5,00 \\
\hline Familia Macrouridae (s/i) & & & 0,16 & 3,33 \\
\hline Caelorinchus sp. & 2,72 & 7,69 & 0,16 & 1,67 \\
\hline Familia Merluccidae (s/i) & 0,68 & 1,92 & 0,73 & 13,33 \\
\hline Merluccius gayi & 60,54 & 78,85 & 4,72 & 60,0 \\
\hline Familia Myctophidae (s/i) & & & 6,27 & 1,67 \\
\hline Hygophum sp. & 12,92 & 3,85 & 2,52 & 1,67 \\
\hline Epigonus crassicaudus & 2,72 & 5,77 & & \\
\hline Helicolenus lengerichi & 2,04 & 5,77 & & \\
\hline Mollusca & 8,16 & 23,08 & 2,43 & 48,33 \\
\hline Clase Cephalopoda (s/i) & & & 0,08 & 1,67 \\
\hline Orden Teuthoidea (s/i) & 0,68 & 1,92 & & \\
\hline Familia Ommastrephidae & & & 0,16 & 3,33 \\
\hline Dosidicus gigas & 7,48 & 21,15 & 1,95 & 40,0 \\
\hline Loligo gahi & & & 0,24 & 3,33 \\
\hline Crustacea & 0,68 & 1,92 & 9,52 & 36,67 \\
\hline Familia Penaeidae & & & 0,24 & 3,33 \\
\hline Familia Benthesicymidae & & & 4,89 & 16,67 \\
\hline Bentheogennema sp. & & & 0,08 & 1,67 \\
\hline Infraorden Caridea & 0,68 & 1,92 & 0,49 & 5 \\
\hline Acanthephyra sp. & & & 1,14 & 6,67 \\
\hline Oplophorus novaezeelandiae & & & 2,60 & 13,33 \\
\hline Familia Gammaridae & & & 0,08 & 1,67 \\
\hline
\end{tabular}

$(*)=52$ estómagos muestreados; $\left({ }^{* *}\right)=60$ estómagos muestreados; $\mathrm{s} / \mathrm{i}=\mathrm{sin}$ identificar

19,52\% vs 90,94\% con ambos tipos de digestión). Finalmente, cuando se compararon las estaciones del año (invierno vs primavera) los valores medios del ISP fueron bajos en ambos casos y similares con un 31,16\% (sólo digestión primaria) contra un 33,29\% (ambos tipos de digestión). En cuanto al valor medio de H, en ambos tipos de digestión, los valores entre sexos fueron muy similares
(2,58 hembras vs 2,37 machos sólo digestión primaria y 2,02 hembras vs 2,03 machos con ambos tipos de digestión). Finalmente, el valor medio del descriptor E resultaron similares para los tipos de digestión (63,33\% hembras vs 59,88\% machos, sólo digestión primaria y $55,91 \%$ hembras vs $56,39 \%$ machos con ambos tipos de digestión). 
Tabla 2. Presas secundarias, con sus valores de los métodos numérico $(N)$ y de frecuencia de ocurrencia $(F)$, presentes en los estómagos de Dosidicus gigas / Secondary prey, with their respectives numeric $(\mathrm{N})$ and frequency of ocurrence (F) values, found in the stomachs of Dosidicus gigas

\begin{tabular}{|c|c|c|c|c|}
\hline Presa secundaria & $\mathrm{N}(\%)$ & $\mathrm{F}(\%)$ & $\begin{array}{l}\text { Presa secundaria } \\
\text { específica }\end{array}$ & $\begin{array}{l}\text { Método de } \\
\text { Identificación }\end{array}$ \\
\hline 1. Infraclase Teleostei (a) & 1,45 & 7,89 & $\begin{array}{l}\text { M. gayi } \\
\text { D. gigas }\end{array}$ & Huesos \\
\hline 2. Orden Stomiiformes (b) & 1,45 & 2,63 & Merluccidae & Neurocráneo \\
\hline 3. Familia Myctophidae (c) & 11,59 & 10,53 & $\begin{array}{l}\text { Teleostei } \\
\text { Merluccidae } \\
\text { Stomias } \text { sp. } \\
\text { M. gayi }\end{array}$ & $\begin{array}{l}\text { Restos cárneos } \\
\text { Fotóforos }\end{array}$ \\
\hline 4. Familia Clupeidae (b) & 0,48 & 2,63 & M. gayi & Espinas \\
\hline 5. Familia Gonostomatidae (b) & 0,97 & 2,63 & M. gayi & Neurocráneo \\
\hline 6. Stomias sp. (b) & 29,47 & 5,26 & $\begin{array}{l}\text { M. gayi } \\
\text { D. gigas }\end{array}$ & Neurocráneo \\
\hline 7. Diaphus sp. (c) & 7,73 & 10,53 & $\begin{array}{l}\text { Merluccidae } \\
\text { Stomias sp. } \\
\text { M. gayi }\end{array}$ & $\begin{array}{l}\text { Fotóforos } \\
\text { Otolitos }\end{array}$ \\
\hline 8. Hygophum sp. (a) & 11,11 & 13,16 & $\begin{array}{l}\text { E. crassicaudus } \\
\text { M. gayi }\end{array}$ & $\begin{array}{l}\text { Fotóforos } \\
\text { Otolitos }\end{array}$ \\
\hline 9. Lampadena sp. (a) & 5,80 & 2,63 & M. gayi & $\begin{array}{l}\text { Fotóforos } \\
\text { Otolitos }\end{array}$ \\
\hline 10. Binghamichthys sp. (b) & 3,38 & 2,63 & M. gayi & Cabeza \\
\hline 11. Merluccius gayi (c) & 2,90 & 10,53 & M. gayi & $\begin{array}{l}\text { Otolitos } \\
\text { Neurocráneo }\end{array}$ \\
\hline 12. Crustacea s/i (a) & 0,97 & 5,26 & M. gayi & Exoesqueleto \\
\hline 13. Euphausiacea (b) & 11,59 & 21,05 & $\begin{array}{l}\text { Stomias sp. } \\
\text { M. gayi }\end{array}$ & Branquias \\
\hline 14. Anomura (b) & 0,48 & 2,63 & Stomias sp. & Exoesqueleto \\
\hline 15. Caridea (b) & 0,48 & 2,63 & Stomias sp. & Exoesqueleto \\
\hline 16. Gammaridae (a) & 0,48 & 2,63 & M. gayi & Exoesqueleto \\
\hline 17. Benthesicymidae (b) & 0,48 & 2,63 & Stomias sp. & Exoesqueleto \\
\hline 18. Pterygosquilla armata (a) & 2,41 & 2,63 & Caelorhinchus sp. & Espinas \\
\hline 19. Pleuroncodes monodon (c) & 3,38 & 7,89 & M. gayi & Exoesqueleto \\
\hline 20. Oplophorus novaezeelandiae (b) & 3,38 & 5,26 & $\begin{array}{l}\text { Merluccidae } \\
\text { Stomias } \text { sp. }\end{array}$ & Exoesqueleto \\
\hline
\end{tabular}

(a): Invierno; (b): Primavera; (c): Ambas estaciones

\section{ANÁLISIS COMPARATIVO DEL ESPECTRO TRÓFICO DE D. GIGAS POR SEXO Y ESTACIÓN}

Cuando se consideró la dieta únicamente la digestión primaria, el sexo resultó ser en general una variable no significativa para explicar las variaciones tróficas en el calamar rojo, tanto en invierno como en primavera (Tabla 3). El ISP de machos y hembras fue alto y similar entre ambas estaciones (ISP invierno $=95,05 \%$; ISP primavera
= 83,14\%). H en la estación de invierno, mostró una alta similitud en la heterogeneidad trófica arrojando también Hmax similares (hembras $=3,70$; machos $=3,58$ ) y E altos y similares. En la primavera, los H, Hmax (hembras = 4,58; machos $=4,52$ ) y E (hembras $=54,73 \%$; machos $=48,50 \%$ ), resultaron similares entre sexos. El $\mathrm{H}$ ponderado (valor que calcula la varianza de $\mathrm{H}$ para toda la muestra en las dos estaciones del año desagregada por sexo), el cual 
Tabla 3. Variación trófica en Dosidicus gigas asociada al sexo, en las estaciones de invierno y primavera, considerando sólo digestión primaria y digestión secundaria / Trophic variation in Dosidicus gigas related to sex, in the winter and spring seasons, being considered just the existence of the primary digestion and the existence of the secondary digestion

\begin{tabular}{|c|c|c|c|c|c|c|c|c|}
\hline \multirow[b]{3}{*}{ Descriptor } & \multicolumn{8}{|c|}{ Considerando a todas las presas como fruto de una digestión primaria } \\
\hline & \multicolumn{4}{|c|}{ Invierno } & \multicolumn{4}{|c|}{ Primavera } \\
\hline & Macho & Hembra & Similitud & $P$ & Macho & Hembra & Similitud & $P$ \\
\hline ISP (\%) & -- & -- & $95,05 \%$ & -- & -- & -- & $83,14 \%$ & -- \\
\hline $\mathrm{H}$ & 2,55 & 2,66 & -- & -- & 2,19 & 2,51 & -- & -- \\
\hline Hmax & 3,58 & 3,70 & -- & -- & 4,52 & 4,58 & -- & -- \\
\hline $\mathrm{E}(\%)$ & 71,25 & 71,93 & -- & -- & 48,50 & 54,73 & -- & -- \\
\hline $\mathrm{Hp}$ & -- & -- & -- & $\mathrm{ns}$ & -- & -- & -- & $*$ \\
\hline \multirow[t]{3}{*}{$\mathrm{F}$} & -- & -- & -- & ns & -- & -- & -- & ns \\
\hline & \multicolumn{8}{|c|}{ Considerando la existencia de la digestión secundaria } \\
\hline & \multicolumn{4}{|c|}{ Invierno } & \multicolumn{4}{|c|}{ Primavera } \\
\hline Descriptor & Macho & Hembra & Similitud & $P$ & Macho & Hembra & Similitud & $P$ \\
\hline ISP (\%) & -- & -- & $98,64 \%$ & -- & -- & -- & $82,57 \%$ & -- \\
\hline $\mathrm{H}$ & 2,09 & 1,85 & -- & -- & 1,98 & 2,21 & -- & -- \\
\hline Hmax & 3,17 & 2,99 & -- & -- & 4,25 & 4,39 & -- & -- \\
\hline E (\%) & 66,06 & 61,58 & -- & -- & 46,72 & 50,23 & -- & -- \\
\hline $\mathrm{Hp}$ & -- & -- & -- & ns & -- & -- & -- & ns \\
\hline $\mathrm{F}$ & -- & -- & -- & ns & -- & -- & -- & $\mathrm{ns}$ \\
\hline
\end{tabular}

* $P<0,05$; ns: no significativa

permitió comparar estadísticamente los valores de $\mathrm{H}$, considerando el número total de ítems presa y la frecuencia de cada uno de ellos, a diferencia del invierno, resultó significativo en la diferenciación de la diversidad alimentaria entre sexos $\left(\mathrm{t}_{0,01(2) 1207}=2,576 ; P<0,05\right)$. Finalmente F, mantuvo la tendencia general de la poca relevancia del sexo, en cuanto a que las diferencias en el número de veces que un determinado ítem presa se encontró en el total de estómagos con contenido entre machos y hembras, no fueron significativas tanto en el invierno como en primavera (Tabla 3).

Cuando la digestión primaria y secundaria fueron consideradas en conjunto, la variable sexo resultó no ser relevante en la explicación de las diferencias dietarias al interior de cada estación del año considerada. En efecto, hubo un alto valor de ISP tanto en invierno (98,64\%), como en primavera (82,57\%) (Tabla 3). El descriptor $\mathrm{H}$ en el invierno fue muy similar entre hembras $(H=1,85)$ y machos $(H=2,09)$, observándose también valores de Hmax similares y de $\mathrm{E}$ altos y similares entre estaciones, tanto en hembras (61,58\%) y machos (66,06\%). A su vez, el H ponderado (calculado sobre el total de individuos de cada sexo) en esta estación, resultó ser no significativo entre $\operatorname{sexos}\left(\mathrm{t}_{0,05(2) 159}=1,96 ; P>0,05\right)$. En la primavera, los valores de $\mathrm{H}$ (hembras = 2,21; machos = 1,98), Hmax y E (hembras = 50,23\%; machos $=46,72 \%$ ), también fueron similares entre sexos. De igual forma, el valor del $\mathrm{H}$ ponderado, no fue significativo $\left(\mathrm{t}_{0,05(2) 160}=1,96 ; P>0,05\right)$, al igual que el valor de F, tanto en el invierno $\left(\chi^{2}=4,2246\right.$; g.l. $=9$; $P>$ $0,05)$, como en la primavera $\left(\chi^{2}=21,7123\right.$; g.l. $=26 ; P>$ $0,05)$.

VARIACIÓN TRÓFICA ESTACIONAL EN D. GIGAS, CONSIDERANDO SOLO LA DIGESTIÓN PRIMARIA

Al comparar las OTUs reconocidas dentro de la estación de invierno $(\mathrm{n}=2)$ respecto de la estación de primavera, así como en contraste entre ambas estaciones sin desagregar la estación de invierno por OTUs, el valor de F fue significativamente distinto entre la OTU1 y la primavera, entre la OTU2 y la primavera y entre el invierno y la primavera (Tabla 4). En cuanto al ISP, y al hacer las mismas comparaciones, se mantuvieron las bajas similitudes al igual que en el escenario donde fue considerada la existencia de la digestión secundaria. Respecto al análisis de los pesos medios de las presas comunes, la OTU2 vs la primavera, en 5 de 7 ítemes presa, mostró diferencias significativas (M. gayi, U= 2082, $P<$ 0,05; Hygophum sp., $\mathrm{U}=465, P<0,05$; Diaphus sp., $\mathrm{U}=$ 
Tabla 4. Variación trófica estacional en Dosidicus gigas, con y sin considerar la digestión secundaria / Seasonal trophic variation of Dosidicus gigas, with or without considering the secondary digestion

\begin{tabular}{llcc}
\hline Descriptor & Comparación & Incluye digestión secundaria & Excluye digestión secundaria \\
\hline $\mathrm{F}$ & OTU1-Primavera & $X^{2}=71,3 ; \mathrm{g} . \mathrm{l} .=27 ; P<0,01$ & $X^{2}=66,8 ; \mathrm{g} . \mathrm{l}=29 ; P<0,01$ \\
& OTU2-Primavera & $X^{2}=120 ; \mathrm{g} . \mathrm{l}=29 ; P<0,01$ & $X^{2}=66,80 ; \mathrm{g} . \mathrm{l}=37 ; P<0,01$ \\
& Invierno-Primavera & $X^{2}=84,3 ; \mathrm{g} . \mathrm{l} .=29 ; P<0,01$ & $X^{2}=66,8 ; \mathrm{g} . \mathrm{l} .=37 ; P<0,01$ \\
& & 16,26 & 15,31 \\
OTU1-Primavera & 41,88 & 39,20 \\
& OTU2-Primavera & 41,72 & 38,96 \\
Invierno-Primavera & OTU2-Primavera & $\mathrm{U}=1970 ; P<0,05$ & $\mathrm{U}=2082 ; P<0,05$ \\
Peso medio1 & OTU2-Primavera & $\mathrm{U}=124 ; P<0,05$ & $\mathrm{U}=465 ; P<0,05$ \\
Peso medio2 & Invierno-Primavera & $\mathrm{U}=1986 ; P<0,05$ & $\mathrm{U}=465 ; P<0,05$ \\
Peso medio3 & Invierno-Primavera & $\mathrm{U}=124 ; P<0,05$ & $\mathrm{Rs}=-1 ; \mathrm{ns}$ \\
Peso medio4 & OTU1-Primavera $(\mathrm{n}=3)$ & $\mathrm{Rs}=0.1 ; \mathrm{ns}$ & $\mathrm{Rs}=0,68 ; P<0,05$ \\
Correlación de Spearmans & OTU2-Primavera $(\mathrm{n}=6)$ & $\mathrm{Rs}=0,5 ; \mathrm{ns}$ & $\mathrm{Rs}=0,56 ; n s$ \\
& Invierno-Primavera $(\mathrm{n}=7)$ & & \\
\hline
\end{tabular}

Peso mediol: Presa en común M. gayi; Peso medio 2: Presa en común Hygophum sp. Peso medio 3: Presa en común M. gayi; Peso medio 4: Presa en común Hygophum sp. $\mathrm{n}=$ número de pares de variables del IIR; ns: no significativa

6, $P<0,05$; Pleuroncodes monodon, $\mathrm{U}=0,00, P<0,05 \mathrm{y}$, Myctophidae, $\mathrm{U}=264, P<0,05) \mathrm{y}$, entre el invierno y la primavera, nuevamente $M$. gayi $(\mathrm{U}=2099, P<0,05)$, al igual que Hygophum sp. (U = 465, $P<0,05)$, Diaphus sp. $(\mathrm{U}=6, P<0,05), P$. monodon $(\mathrm{U}=0, P<0,05) \mathrm{y}$ Myctophidae ( $U=264, P<0,05)$, mostraron diferencias significativas en sus pesos medios, en 5 de 7 ítemes presa en común. Finalmente, no hubo una correlación significativa entre la OTU1 y la primavera (Tabla 4). En el caso de la OTU2 y la primavera, la correlación resultó positiva y significativa y, entre el invierno y la primavera, la asociación no fue significativa y muy similar cuando se consideró la digestión secundaria.

\section{VARIACIÓN TRÓFICA ESTACIONAL EN D. GIGAS ASOCIADA AL TAMAÑO}

En invierno, cuando sólo fue considerada la digestión de tipo primaria el ISP de la clase 1 mostró una similitud trófica baja en relación al resto de las clases (ISP promedio $=33,21 \%$ ), lo mismo que la clase 2 (ISP promedio $=2,39 \%$ ) (Tabla 5). Las clases 3, 4, 5 y 6 presentaron una alta similitud (ISP > 69,60; promedio $=82,13 \%$ ), apoyando también la existencia de, al menos, 2 grupos discretos. Dada la baja similitud alimentaria de la clase 1 con las de mayor tamaño y el hecho de que parte de la bibliografía reconoce a estas dos primeras clases conformadas por individuos entre los 52 y los $70 \mathrm{~cm}$ (LM) como parte de un solo grupo, se optó por considerar a estos individuos como una sola OTU, determinando así la existencia de 2 grupos discretos, la OTU1 compuesta por las clases de tamaño 1 y 2 y la OTU2, compuesta por las clases de tamaño 3, 4, 5 y 6. La unión de las clases de LM 1 y 2 también ayudó a disminuir en parte el problema del bajo tamaño de muestra obtenido para ambas clases.

Para la estación de primavera, sobre la base de las 5 clases de LM constituidas la clase 1 mostró un ISP con una relativa alta similitud trófica con la clase 2 (ISP = $55,13 \%)$, pero inferior y diferente con las restantes 3 clases de tamaño (ISP $<43,45 \%$; promedio $=36,95 \%$ ). En cambio la clase 3 y restantes presentaron una alta similitud trófica (ISP > 59,54\%; promedio = 66,18\%) (Tabla 5).

Al considerar la digestión primaria y secundaria, en la estación de invierno, y sobre la base de las 6 clases de tamaños previamente conformadas, el análisis comparativo del ISP (Tabla 5), estableció una clara diferenciación alimentaria entre las clases de tamaño 1 y 2 con el resto (ISP promedio $=19,52 \%$ ) y una alta similitud trófica entre las clases de tamaño 3, 4, 5 y 6 (ISP promedio $=90,94 \%$ ). Hubo una baja similitud entre las clases de LM 1 y 2, ello podría deberse al bajo número de individuos recolectados pertenecientes a ambas clases $(n=5)$ (Tabla 5). Por su parte, en primavera, sobre la base de 5 clases de LM 
Tabla 5. Indice de similitud porcentual (ISP, \%) de la dieta de Dosidicus gigas entre clases de longitud del manto (LM), considerando o no la digestión la digestión secundaria / Percentage of similarity index (ISP, \%) of Dosidicus gigas diet among class size (mantle length, LM), with or without considering the secondary digestion

\begin{tabular}{|c|c|c|c|c|c|c|c|c|c|}
\hline \multirow{3}{*}{$\begin{array}{c}\text { Clases de } \\
\text { LM }\end{array}$} & \multicolumn{9}{|c|}{ Considerando sólo la existencia de la digestión primaria } \\
\hline & \multicolumn{5}{|c|}{ Invierno } & \multicolumn{4}{|c|}{ Primavera } \\
\hline & 2 & 3 & 4 & 5 & 6 & 2 & 3 & 4 & 5 \\
\hline 1 & 16,72 & 35,18 & 39,07 & 35,83 & 39,25 & 54,70 & 41,28 & 28,46 & 43,45 \\
\hline 2 & --- & 0,51 & 3,30 & 1,16 & 4,58 & --- & 70,75 & 59,54 & 68,25 \\
\hline 3 & & --- & 91,40 & 87,45 & 69,60 & & --. & 65,08 & 89,32 \\
\hline 4 & & & --- & 90,22 & 75,14 & & & --- & 64,04 \\
\hline 5 & & & & --- & 78,98 & & & & --- \\
\hline
\end{tabular}

\begin{tabular}{|c|c|c|c|c|c|c|c|c|c|}
\hline \multirow{3}{*}{$\begin{array}{c}\text { Clases de } \\
\text { LM }\end{array}$} & \multicolumn{9}{|c|}{ Considerando la existencia de la digestión secundaria } \\
\hline & \multicolumn{5}{|c|}{ Invierno } & \multicolumn{4}{|c|}{ Primavera } \\
\hline & 2 & 3 & 4 & 5 & 6 & 2 & 3 & 4 & 5 \\
\hline 1 & 16,72 & 35,27 & 37,8 & 35,02 & 39,91 & 55,13 & 43,45 & 25,01 & 42,40 \\
\hline 2 & --- & 0,60 & 1,98 & 0,35 & 5,24 & -- & 72,20 & 56,85 & 67,25 \\
\hline 3 & & -- & 94,86 & 91,88 & 85,28 & & --- & 65,26 & 87,28 \\
\hline 4 & & & -- & 92,22 & 88,84 & & & -- & 63,92 \\
\hline 5 & & & & --- & 92,58 & & & & -- \\
\hline
\end{tabular}

establecidas, la clase 1 mostró una relativa alta similitud con la clase 2 (ISP $=55,13 \%$ ), pero baja con las restantes clases (ISP < 43,45\%). Sin embargo, se observó una alta similitud entre las 4 clases mayores (ISP > 56,85\%; promedio $=65,43 \%)$.

\section{VARIACIÓN TRÓFICA ESTACIONAL EN D. GIGAS, CONSIDERANDO SOLO LA DIGESTIÓN SECUNDARIA}

Conforme los resultados obtenidos anteriormente con las variables sexo (no significativa) y tamaño (reconocimiento de OTU1 y OTU2 en invierno y ninguna en primavera), se realizó el análisis comparativo estacional. El valor de $\mathrm{F}$ fue significativamente distinto entre la OTU1 y la primavera $\left(\chi^{2}=71,32\right.$; g.l. $\left.=27 ; P<0,005\right)$; entre la OTU2 y la primavera $\left(\chi^{2}=120,4078\right.$; g.l. $\left.=29 ; P<0,005\right)$ y, entre el invierno y la primavera $\left(\chi^{2}=84,2656\right.$; g.l. $\left.=29 ; P<0,005\right)$ (Tabla 5). El ISP por otra parte, mostró baja similitud en cada una de las comparaciones (OTU1 vs primavera, ISP $=16,26 \%$; OTU2 vs primavera, ISP $=41,88 \%$ e, invierno vs primavera, ISP $=41,72 \%)$. En los casos donde fue posible realizar la comparación de los pesos medios de las presas comunes entre estaciones, la OTU2 respecto de la primavera, en 2 de 4 ítemes presa comunes, mostraron diferencias significativas ( $M$. gayi, $\mathrm{U}=1970, P<0,05 \mathrm{e}$ Hygophum sp., $\mathrm{U}=124, P<0,05)$, destacando la importancia que tiene $M$. gayi como presa, con un IIR superior al 90\%. El análisis estacional, considerando al invierno no desagregado en sus dos OTUs sino en forma completa, volvió a consignar los mismos resultados, $M$. gayi, la presa más importante $(\mathrm{U}=1986, P<0,05)$ e Hygophum sp. ( $\mathrm{U}=124, P<0,05)$, mostraron nuevamente diferencias significativas respecto de la primavera. Finalmente, el coeficiente de Spearman mostró una correlación negativa aunque no significativa entre los valores del IIR de las presas comunes entre la OTU1 y la primavera (Rs $=-1 ; P>0,05 ; \mathrm{n}=3$ ). Entre la OTU2 y la primavera, si bien dicha correlación fue positiva y alta, no fue significativa (Rs = 0,6; $P>0,05 ; \mathrm{n}=6$ ) y, entre el invierno y la primavera, el grado de correlación fue positivo pero no significativo (Rs $=0,5 ; P>0,05 ; \mathrm{n}=7$ ).

\section{Discusión}

Los estudios basados en la conducta alimentaria de las especies otorgan elementos básicos para comprender su ecología trófica. Así, la determinación de la digestión secundaria puede contribuir a establecer en forma más real el espectro trófico. Sin embargo, en los escasos trabajos donde se reconoce la existencia de la digestión secundaria, aquellos ítemes presa señalados como 'presas de presas', han sido eliminados de los datos a priori, usando como criterio la exclusión de presas con valores bajo el 1\% del IIR (Clarke \& Paliza 2000, Markaida 2001), 
aunque no hay referencias de cómo o por qué esos ítemes fueron determinados como presas secundarias.

Bajo el marco de esta investigación, que determina $a$ posteriori la existencia y relevancia de la digestión secundaria, fue necesario reconocer los sesgos de interpretación en que se podrían incurrir, ya que el uso de redes de arrastre para la toma de muestras de los calamares puede inducir a una posible sobreestimación de la importancia relativa de algunos ítemes presa (Field et al. 2007, Ibáñez et al. 2008). La merluza común puede ser un buen ejemplo, asociado a la conducta oportunista del calamar rojo, el cual tiende a atacar presas abundantes bajo circunstancias favorables como las agregaciones de los cardúmenes durante los lances de pesca (Sánchez 2003, Field et al. 2007). Sin embargo, lo anterior no sería incidente para esta investigación, ya que los dos escenarios bajo comparación (con y sin la consideración de la digestión secundaria), estaban sujetos a la misma situación de sobreoferta alimentaria, pero del mismo recurso. Además, del punto de vista cuantitativo los resultados mostraron mínimas diferencias, incluyendo la significancia de las mismas, cuando se consideraron ambos tipos de digestión con respecto a la digestión primaria (Tablas 3, 4 y 5).

El sexo de los calamares no fue relevante para explicar diferencias dietarias, tal como ha sido evidenciado por otros autores (Roper et al. 1984, Erhardt 1991, Wormuth 1998, Clarke \& Paliza 2000, Nigmatullin et al. 2001, Markaida \& Sosa-Nishizaki 2003), inclusive en los casos en que la digestión secundaria fue considerada en el análisis (Markaida 2001). En general estos autores postulan que las áreas geográficas y la estacionalidad son más relevantes que el sexo en la variación alimentaria del calamar rojo. En otros grandes depredadores como el pez espada (Xiphias gladius), también se ha observado la escasa influencia del sexo en la dieta de este pez, aunque no se detallan explicaciones al respecto (Letelier et al. 2009). El alto porcentaje de presas compartidas entre machos y hembras adultos pudo obedecer a un comportamiento alimentario generalista de Dosidicus gigas. En efecto, ha postulado que esta especie se alimenta indiscriminadamente de cualquier presa que se mueva, si es abundante y de tamaño adecuado (Nesis 1970). Lo anterior, junto con el hecho de que los cardúmenes de adultos están formados por machos y hembras de tamaños similares, posibilitaría esta uniformidad en la dieta sin importar el sexo.

Por otro lado, los resultados del presente estudio mostraron que la estacionalidad (invierno vs primavera) fue influyente en los hábitos tróficos del calamar. Junto al factor de la estacionalidad, la diversidad trófica entre sexos cuando no se consideró la digestión secundaria, mostró diferencias significativas en la estación de primavera, debido al mayor espectro trófico de las hembras, producto de la presencia de 'presas de las presas' consideradas artificialmente como 'presas'. Este artefacto (mayor variabilidad en las 'presas de presas' en las hembras consideradas erróneamente como 'presas'), podría explicarse por la mayor ocurrencia de estómagos llenos en éstas, a causa de su mayor tamaño, lo que aumenta la probabilidad (por el mayor número) de considerar 'presas de presas' como 'presas'.

Si bien la digestión secundaria tuvo poco efecto en la importancia relativa de los nuevos ítemes presa incorporados al espectro alimentario del calamar rojo, así como en modificar la importancia de las presas fruto de la digestión primaria, tal tipo de digestión puede jugar un rol importante en la heterogeneidad del espectro trófico entre machos y hembras, especialmente en aquellas estaciones del año, en donde se daría una mayor oferta alimentaria disponible o mayor disposición de presas alternativas o potenciales (Markaida 2001, Markaida et al. 2008). Bajo tal escenario, estos hallazgos habrían sugerido erróneamente, al no considerar la digestión secundaria, que la mayor oferta alimentaria disponible en la estación de primavera habría sido mejor aprovechada por las hembras, debido al mayor numero de 'presas de presas' consideradas como 'presas’ en comparación con los machos.

En cuanto a la variación alimentaria en el calamar rojo, asociada al tamaño y, a la determinación de la existencia de las OTUs, se observó gran similitud al comparar por separado cada una de las estaciones (invierno y primavera), es decir, cuando se incluyó y excluyó la digestión secundaria. Los descriptores F e ISP, al ser analizados estacionalmente mostraron básicamente los mismos resultados al incluir y excluir la digestión secundaria, ya sea en términos de los valores obtenidos o de su significancia estadística (Tabla 4). Lo anterior implica que la digestión secundaria, posee un efecto sólo cualitativo, sobreestimando los ítems presas de la especie bajo estudio, pero no en la importancia relativa de las presas (efecto cuantitativo), ya que es enmascarada por el bajo IIR, dado por la baja frecuencia de ocurrencia o por un escaso valor volumétrico de las presas secundarias.

La dieta del calamar rojo también fue similar en cada estación (invierno y primavera) cuando en cada una de 
ellas se compararon los descriptores tróficos en relación a los dos escenarios bajo análisis (digestión primaria vs digestión primaria y secundaria), considerando la variable sexo (Tabla 3). En ambas situaciones, los hábitos tróficos del calamar fueron significativamente distintas entre estaciones, en el descriptor $\mathrm{F}$, en los valores de $\mathrm{U}$ y en los valores de Rs (salvo la OTU2 vs primavera), siendo esto concordante con otros trabajos asociados al comportamiento alimentario estacional de Dosidicus gigas (Ehrhardt 1991). Resultados similares han sido reportados en otros depredadores grandes, como es el caso del pez espada Xiphias gladius (Ibáñez et al. 2004). Así, en la comparación de los pesos medios entre las presas comunes, la diferencia no estuvo centrada en eventuales variaciones en dichos pesos, sino en la aparición (cuando no es considerada la digestión de tipo secundaria) de nuevos ítemes presa comunes a ambas estaciones, tal como se observó en la comparación entre la OTU2 y la primavera, así como entre la estación de invierno sin desagregar por OTUs y la primavera (P. monodon, Diaphus sp. y la familia Myctophidae). La baja similitud trófica estacional es concordante con aquellos trabajos que han postulado que la estacionalidad sería relevante en los cambios de la dieta de los grandes predadores por causa de la mayor abundancia u oferta alimentaria (Ibáñez et al. 2004, Letelier et al. 2009). Sin embargo, Chong et al. (2005) no encontraron diferencias estacionales en la dieta del calamar rojo, cuando hicieron comparaciones de la frecuencia de ocurrencia de presas, aunque hubo una clara diferencia estacional en la importancia relativa de algunos grupos de presa entre invierno y primavera, así como diferencias cualitativas importantes en la composición de los peces como ítemes dietarios. De esta forma, se plantea que las diferencias dietarias estacionales encontradas en nuestro trabajo para el calamar rojo, obedecen fundamentalmente a la mayor oferta alimentaria disponible en la primavera, la que es aprovechada en forma similar por machos y hembras vinculados a las mismas OTUs. La mayor oferta alimentaria disponible en primavera, pudo ser evidenciada cuando se efectuó el cálculo del Î́ndice de Selectividad (SEL) para ponderar la preferencia por un determinado tipo de presa, ya que tal índice exige el porcentaje en número del alimento potencialmente disponible en el ambiente (resultados no incluidos en este trabajo).

La baja influencia que tuvo la digestión secundaria en el análisis cuantitativo de la dieta del calamar rojo, se debe a su escaso aporte, con un IIR no superior al $1 \%$. Esto es concordante con lo señalado por otros autores para la misma especie (Markaida 2001, Markaida \& SosaNishizaki 2003), quienes indican que aquellas presas con un IIR igual o inferior al 1\% no juegan un rol relevante en la alimentación del calamar rojo, más allá del valor gráfico irrelevante que poseen presas con estos valores. Además no solamente por su bajo impacto cuantitativo y energético, sino también porque estas presas podrían estar asociadas a una o más de sus presas primarias, siendo más relevante este tipo de digestión en la transmisión de parásitos (Shukhgalter \& Nigmatullin 2001, Ibáñez et al. 2008, Pardo-Gandarillas et al. 2004, 2007b, 2009, Nigmatullin et al. 2009), lo cual también ha sido descrito para otras especies de calamares pertenecientes a la familia Ommastrephidae, tales como Illex coindetii (González et al. 2003) y Ommastrephes bartramii (Nigmatullin et al. 2009).

Sin embargo, el análisis comparativo, desde un punto de vista cualitativo en términos de riqueza de los ítemes presa y amplitud del espectro trófico del calamar rojo, reveló la importancia que reviste la digestión secundaria si esta no es considerada. El efecto principal de este hecho, se observa en la caracterización del espectro trófico estacional del calamar rojo. De no haber existido un análisis previo de la existencia de la digestión secundaria, hubiéramos erróneamente señalado (considerando solo las presas identificadas en los niveles taxonómicos más bajos) como parte del espectro trófico invernal de Dosidicus gigas en la zona Centro-Sur de Chile, incluyendo algunos casos como nuevos ítems presa no descritos para este depredador a Lampadena sp., Diaphus sp., Pterygosquilla sp., Pleuroncodes monodon y los anfípodos gamáridos. Por su parte en la estación de primavera habríamos mencionado a Euphausiacea, Binghamichthys sp., Diaphus sp. y P. monodon como presas del calamar rojo, en consecuencia que fueron 'presas de las presas'. Otro ejemplo se dio en términos de que si bien se mantuvo la predominancia en ambos escenarios de un mayor número de ítemes presa en la estación de primavera respecto del invierno, claramente hubo una importante reducción del número de éstos, cuando la digestión secundaria fue considerada y en consecuencia, todas las 'presas de presas' fueron descartadas del análisis (Tablas 1 y 2). Este hecho se constituyó en un factor relevante, pues a lo menos entra a cuestionar qué tan importante es el aumento del espectro trófico de Dosidicus gigas, con el cambio estacional. Una situación similar reportan Markaida \& Sosa-Nishizaki (2003) quienes observaron un menor número de ítemes presas, para una misma localidad, en la estación de invierno respecto de la primavera. El mecanismo de 
descarte de presas con un IIR $<1 \%$ si bien tiene un propósito práctico dada la escasa relevancia que poseen en los análisis tróficos de tipo cuantitativos para la especie (Markaida 2001, Markaida \& Sosa-Nishizaki 2003), la alta similitud en el bajo valor del índice con los resultados permiten inferir que hay una alta probabilidad de que parte importante de lo que estos autores descartaron, sean presas asociadas a la digestión secundaria. A esto se debe agregar el reconocimiento explícito que se hace de la existencia de la digestión secundaria para esta especie, particularmente en individuos con LM superior a los 15 $\mathrm{cm}$ y que forman parte de las denominadas presas poco frecuentes, transitorias o raras (Markaida 2001).

También resulta relevante hacer notar que algunas presas encontradas en los estómagos del calamar rojo fueron reconocidas en algunos casos como parte de la digestión primaria y en otros de tipo secundaria (i.e., Merluccius gayi, Pleuroncodes monodon, Hygophum sp., Stomias sp., Oplophorus novaezeelandiae). Si bien este hecho puede resultar algo esperable, ejemplifica de buen modo la complejidad de las relaciones tróficas entre las especies, donde se pueden inferir entre otras, relaciones de canibalismo y del fuerte dinamismo en la posición que ocupan los organismos en los distintos niveles tróficos. Polo-Silva et al. (2007) señalaron que estos estudios pueden ser de gran utilidad en el conocimiento del flujo energético en las comunidades marinas y en las interacciones entre las especies a nivel de competencia y depredación.

La digestión secundaria sólo tuvo un rol importante en la caracterización cualitativa de la biología trófica de esta especie. En consecuencia, el aporte en considerar su existencia es que permite 'calibrar' o 'ajustar' las presas realmente consumidas por el calamar, reflejando de mejor forma su posición en redes tróficas de las cuales forma parte, especialmente en su papel de depredador. Tras el ajuste efectuado por la digestión secundaria, se sigue ratificando lo señalado por Amaratunga (1983), en términos del rol fundamental que juegan los cefalópodos (ejemplificado con D. gigas) en las redes tróficas marinas, al abarcar varios niveles en la transmisión de la energía y en que de acuerdo a este estudio, D. gigas ocuparía un nicho de consumidor de tercer a cuarto orden trófico.

\section{Agradecimientos}

Los autores agradecen la colaboración prestada en la toma de muestras de calamar rojo, a las empresas pesqueras El Golfo S.A. y Congelados Pacífico S.A., así como a los profesores Hugo I. Moyano y la Dra. Margarita Marchant de la Universidad de Concepción, por la atención prestada durante la realización de este trabajo, por sus comentarios, sugerencias y colaboración en el análisis de las muestras.

\section{LITERATURA CITADA}

Amaratunga T. 1983. The rol of cephalopods in the marine ecosistema. FAO Fisheries Technical Paper 231: 379-415.

Argüelles J, P Rodhouse, P Villegas \& G Castillo. 2001. Age, growth and population structure of the jumbo flying squid Dosidicus gigas in Peruvian waters. Fisheries Research 54: 51-61.

Bahamonde N. 1968. Bosquejo taxonómico sobre los estomatópodos de Chile. Revista Universitaria 31: 107-120.

Bazzino G, C Salinas \& U Markaida. 2007. Variabilidad en la estructura poblacional del calamar gigante (Dosidicus gigas) en Santa Rosalía, región central del Golfo de California. Ciencias Marinas 33(2): 173-186.

Berg J. 1979. Discussion of methods of investigating the food of fishes, with reference to a preliminary study of the prey of Gobiusculus flavescens (Gobiidae). Marine Biology 50: 263-273.

Bloom S. 1981. Similarity indices in community studies: potential pitfalls. Marine Ecology Progress Series 5(2): 125128.

Bojórquez E, S Martínez, F Arreguín \& M Nevárez. 2001. Estimations of catchability at length for the jumbo squid (Dosidicus gigas) fishery in the Gulf of California, Mexico. CalCOFI Reports 42: 167-171.

Bowman T \& L Abele. 1982. Classification of the recent Crustacea. In: Bliss D \& L Abele (eds). Systematics, the fossil record, and biogeography. The biology of Crustacea, pp. 1-27. Academic Press, New York.

Brunetti N, M Ivanovic \& M Sakai. 1999. Calamares de importancia comercial en la Argentina. Biología, distribución, pesquerías, muestreo biológico, 45 pp. Instituto Nacional de Investigación y Desarrollo Pesquero, Secretaría de Agricultura, Ganadería, Pesca y Alimentación, Mar del Plata.

Chong J, C Oyarzún, R Galleguillos, E Tarifeño, R Sepúlveda \& C Ibáñez. 2005. Parámetros biológicopesqueros de la jibia Dosidicus gigas (Orbigny, 1835) (Cephalopoda: Ommastrephidae), frente a la costa de Chile central (29ㅇ-40 $)$ durante 1993-1994. Gayana 69(2): 319328.

Cid L, M Valenzuela \& A Mora. 1990. Inferencia estadística, 319 pp. Universidad de Concepción, Concepción.

Clarke MR. 1997. Cephalopods in the stomach of a sperm whale stranded between the islands of Terschelling and Ameland, southern North Sea. Biologie 67: 53-55.

Clarke MR \& P Pascoe. 1997. Cephalopods species in the diet of a sperm whale (Physeter catodon) stranded at Penzance, Cornwall. Journal of the Marine Biological Associated of the United Kingdom 77: 1255-1258. 
Clarke M, N Macleod \& O Paliza. 1976. Cephalopod remains from the stomachs of sperm whales caught off Peru and Chile. Journal of Zoology 180: 477-493.

Clarke MR, M Martins \& P Pascoe. 1993. The diet of sperm whales (Physeter macrocephalus Linnaeus 1758) off the Azores. Philosophical Transactions of the Royal Society of London, B 339: 67-82.

Clarke R \& O Paliza. 2000. The Humboldt current squid Dosidicus gigas (Orbigny, 1835). Revista de Biología Marina y Oceanografía 35(1): 1-39.

Clarke R, O Paliza \& A Aguayo. 1988. Sperm whales of the Southeast Pacific. Part IV. Fatness, food and feeding. Investigations on Cetacea 21: 53-195.

Cohen D, T Inada, T Iwamoto \& N Scialabba. 1990. FAO species catalogue. Volume 10. Gadiform fishes of the world (order Gadiformes). An annotated and illustrated catalogue of cods, hakes, grenadiers fishes known to date. FAO Fisheries Synopsis 10(125): 1-442.

Crespin de Billy V, S Doledec \& D Chessel. 2000. Biplot presentation of diet composition data: an alternative for fish stomach contents analysis. Journal of Fish Biology 56: 961-973.

Cubillos L, C Ibáñez, C González \& A Sepúlveda. 2004. Pesca de Investigación: Pesca de jibia (Dosidicus gigas) con red de cerco entre la V y X regiones, año 2003. Informe Final, Instituto de Investigaciones Pesqueras Octava Región, Talcahuano, 48 pp.

Ehrhardt N. 1991. Potential impact of a seasonal migratory jumbo squid (Dosidicus gigas) stock on a Gulf of California sardine (Sardinops sagax caerulea) population. Bulletin of Marine Science 49(1-2): 325-332.

Falabella F, R Meléndez \& M Vargas. 1995. Claves osteológicas para peces de Chile central, un enfoque arqueológico, 208 pp. Editotrial Artegrama, Santiago.

Fernández F \& J Vásquez. 1995. La jibia gigante Dosidicus gigas (Orbigny, 1835) en Chile: Análisis de una pesquería efímera. Estudios Oceanológicos 14: 17-21.

Field J, W Walker \& A Jason. 2007. Range expansion and trophic interactions of the jumbo squid, Dosidicus gigas, in the California Current. CalCOFI Reports 48: 131-146.

Finke D \& W Snyder. 2008. Niche partitioning increases resource exploitation by diverse communities. Science 321: 1488-1490.

González A, S Pascual, C Gestal, E Abollo \& A Guerra. 2003. What makes a cephalopod a suitable host for parasite? The case of Galician waters. Fisheries Research 60: 177-183.

González P \& J Chong. 1997. Alimentación del lenguado de ojos chicos Paralichthys microps (Günter, 1881) (Pleuronectiformes, Paralichthydae) en Bahía Concepción. (VIII Región, Chile). Gayana Zoología 61(1): 7-13.

González P \& J Chong. 2006. Biología reproductiva de Dosidicus gigas D’Orbigny 1835 (Cephalopoda, Ommastrephidae) en la zona Norte-Centro de Chile. Gayana 70(2): 237-244.
Granadeiro J \& M Silva. 2000. The use of otoliths and vertebrae in the identification and size-estimation of fish in predator-prey studies. Cybium 24(4): 383-393.

Hoffman M. 1979. The use of Pielou's method to determine sample size in food studies. In: Lipovsky J \& CA Simenstad (eds). Gutshop' 78, Fish food habits studies. Proceeding of the Second Pacific Northwest Technical Workshop, October 10-13, 1978, Maple Valley, pp. 56-61. University of Washington, Washington Sea-Grant Publications Seattle, Washington.

Hyslop E. 1980. Stomach contents analysis - a review of methods and their application. Journal of Fish Biology 17: 411-429.

Ibáñez C, C González \& L Cubillos. 2004. Dieta del pez espada Xiphias gladius, 1758, en aguas oceánicas de Chile central en invierno de 2003. Investigaciones Marinas 32(2): 113-120.

Ibáñez C, H Arancibia \& L Cubillos. 2008. Biases in determining the diet of jumbo squid Dosidicus gigas (D’Orbigny 1835) (Cephalopoda: Ommastrephidae) off southern-central Chile $\left(34^{\circ} \mathrm{S}-40^{\circ} \mathrm{S}\right)$. Helgoland Marine Research 62(4): 331-338.

Jobling M \& A Breiby. 1986. The use and abuse of fish otoliths in studies of feeding habits of marine piscivores. Sarsia 71: 265-274.

Krebs Ch. 1985. Ecología. Estudio de la distribución y la abundancia, 753 pp. Editorial Harla, México.

Labbé J \& P Arana. 2001. Alimentación de orange roughy, Hoplostethus atlanticus (Pisces: Trachichthyidae), en el Archipiélago de Juan Fernández, Chile. Revista de Biología Marina y Oceanografía 36: 75-82.

Leible M \& P Miranda. 1989. El otolito sagitta en el reconocimiento de diferentes especies de teleósteos de la costa central de Chile. Boletín de la Sociedad de Biología de Concepción 60: 149-160.

Letelier S, R Meléndez, E Carreño, S Lopez \& P Barria. 2009. Alimentación y relaciones tróficas del pez espada (Xiphias gladius Linnaeus, 1758), frente a Chile centronorte durante 2005. Latin American Journal of Aquatic Research 37(1): 107-119.

Lotka A. 1925. Elements of physical biology, 465 pp. Williams and Wilkins, Baltimore.

Markaida U. 2001. Biología del calamar gigante Dosidicus gigas Orbigny, 1835 (Cephalopoda: Ommastrephidae) en el Golfo de California, México. Tesis Doctoral, Centro de Investigación Científica y de Educación Superior de Ensenada, Ensenada, México, 387 pp.

Markaida U. 2006. Food and feeding of jumbo squid Dosidicus gigas in the Gulf of California and adjacent waters after the 1997-98 El Niño event. Fisheries Research 79: 16-27.

Markaida U \& O Sosa-Nishizaki. 2003. Food and feeding habits of jumbo squid Dosidicus gigas (Cephalopoda: Ommastrephidae) from the Gulf of California, México. Journal of the Marine Biological Association of the United Kingdom 83(3): 507-522. 
Markaida U, W Gilly, C Salinas \& J Ashley. 2008. Food and feeding of jumbo squid Dosidicus gigas in the Central Gulf of California during 2005-2007. CalCOFI Reports 49: 90-103.

Méndez M. 1981. Claves de identificación y distribución de los langostinos y camarones (Crustacea: Decapoda) del mar y ríos de la costa del Perú. Boletín del Instituto del Mar del Perú 5: 1-170.

Moreno C. 2001. Métodos para medir la biodiversidad, 84 pp. M \& T - Manuales y Tesis SEA, Cyted, Zaragoza.

Nelson J. 1994. Fishes of the world, 600 pp. John Wiley \& Sons, New York.

Nesis K. 1970. The biology of the giant squid of Peru and Chile, Dosidicus gigas. Okeanologiya 10(1): 108-118.

Nigmatullin Ch, K Nesis \& A Arkhipkin. 2001. A review of the biology of the jumbo squid Dosidicus gigas (Cephalopoda: Ommastrephidae). Fisheries Research 54: 9-19.

Nigmatullin Ch, A Shchetinnikov \& O Shukhgalter. 2009. On feeding and helminth fauna of neon flying squid Ommastrephes bartramii (Lesueur, 1821) (Cephalopoda: Ommastrephidae) in the southeastern Pacific. Revista de Biología Marina y Oceanografía 44(1): 227-235.

Pardo-Gandarillas M, F Garcías \& M George-Nascimento. 2004. La dieta y fauna de endoparásitos del pejesapo Gobiesox marmoratus Jenyns, 1842 (Pisces: Gobiesocidae) en el litoral central de Chile están conectadas pero no correlacionadas. Revista Chilena de Historia Natural 77: 627-637.

Pardo-Gandarillas M, F Duarte, J Chong \& C Ibáñez. 2007a. Dieta de tiburones juveniles Prionace glauca (Linnaeus, 1758) (Carcharhiniformes: Carcharhinidae) en la zona litoral centro-sur de Chile. Revista de Biología Marina y Oceanografía 42(3): 365-369.

Pardo-Gandarillas M, K González, C Ibáñez \& M GeorgeNascimento. 2007b. Parasites of two deep-sea fish Coelorhynchus chilensis (Pisces: Macrouridae) and Notacanthus sexspinis (Pisces: Notacanthidae) from Juan Fernández Archipiélago, Chile. JMBA2-Biodiversity Records: 1-5.

Pardo-Gandarillas M, K Lohrmann, A Valdivia \& C Ibáñez. 2009. First record of parasites of Dosidicus gigas (d’Orbigny, 1835) (Cephalopoda: Ommastrephidae) from the Humboldt Current system off Chile. Revista de Biología Marina y Oceanografía 44(2): 397-408.

Polo-Silva C, A Baigorrí-Santacruz, F Galván-Magaña, M Grijalba-Bendeck \& A Sanjuan-Muñoz. 2007. Hábitos alimentarios del tiburón zorro Alopias superciliosus (Lowe, 1839), en el Pacífico ecuatoriano. Revista de Biología Marina y Oceanografía 42(1): 59-69.

Retamal M. 1977. Los crustáceos decápodos chilenos de importancia económica. Gayana Zoología 39: 1-48.
Retamal M. 1981. Catálogo ilustrado de los crustáceos decápodos de Chile. Gayana Zoología 44: 1-110.

Rojo A. 1976. Osteología de la merluza argentina (Merluccius hubbsi, Marini 1933). Boletín del Instituto Español de Oceanografía 219: 1-61.

Roper C, R Young \& G Voss. 1969. An illustrated key to the families of the order Teuthoidea (Cephalopoda). Smithsonian Contributions to Zoology 13: 1-32.

Roper C, M Sweeney \& C Nauen. 1984. FAO Species catalogue. Cephalopods of the world. An annotated and illustrated catalogue of species of interest to fisheries. FAO Fisheries Synopsis 125(3): 1-277.

Ruiz V, C Oyarzún \& S Gacitúa. 1995. Osteología de Macrourus holotrachys Gunther, 1878 (Pisces, Gadiformes, Macrouridae). Boletín de la Sociedad de Biología de Concepción 66: 125-140.

Ruiz-Cooley R, D Gendron, S Aguíñiga, S Mesnick \& J Carriquiry. 2004. Trophic relationships between sperm whales and jumbo squid using stable isotopes of $\mathrm{C}$ and $\mathrm{N}$. Marine Ecology Progress Series 277: 275-283.

Sáez L. 1982. Myctophidae (Pisces, Osteichthyes, Myctophiformes) recolectado por la expedición 'ItzumiPelágico I’. Enero-Febrero 1980. Tesis de Biólogo Marino, Universidad de Concepción, Concepción, 100 pp.

Sánchez P. 2003. Cephalopods from off the Pacific coast of Mexico: biological aspects of the most abundant species. Scientia Marina 67(1): 81-90.

Santos M, M Clarke \& G Pierce. 2001. Assessing the importance of cephalopods in the diet of marine mammals and other top predators: problems and solutions. Fisheries Research 52: 121-139.

Shukhgalter O \& Ch Nigmatullin. 2001. Parasitic helminths of jumbo squid Dosidicus gigas (Cephalopoda: Ommastrephidae) in open waters of the central east Pacific. Fisheries Research 54: 95-110.

Smith S \& H Whitehead. 2000. The diet of Galapagos sperm whales Physeter macrocephalus as indicated by fecal sample analysis. Marine Mammal Science 16(2): 315-325.

Soberon J \& M Nakamura. 2009. Niches and distributional areas: concepts, methods, and assumptions. Proceedings of the National Academy of Sciences 108(40): 1-7.

Taipe A, C Yamashiro, L Mariátegui, P Rojas \& C Roque. 2001. Distribution and concentrations of jumbo flying squid (Dosidicus gigas) off the Peruvian coast between 1991 and 1999. Fisheries Research 54: 21-32.

Ulloa P, M Fuentealba \& V Ruiz. 2006. Hábitos alimentarios de Dosidicus gigas (D’Orbigny, 1835) (Cephalopoda: Teuthoidea) frente a la costa centro-sur de Chile. Revista Chilena de Historia Natural 79: 475-479.

Volterra V. 1926. Variazioni e fluttuazioni del numero d'individui in specie animali conviventi. Men Ray Accademia Nazionale dei Lincei, Roma 2: 31-113. 
Wiff R \& R Quiñones. 2004. Parametrización ambiental en modelos biológico-pesqueros. Una revisión. Gayana 68(1): 76-92.

Wisner R. 1971. Descriptions of eight new species of myctophid fishes from the eastern Pacific Ocean. Copeia 1: 39-54.

Wisner R. 1974. The taxonomy and distribution of lanterfishes (Family Myctophidae) of the eastern Pacific Ocean. Navy Ocean Research and Development Activity (Norda) Report 3, St. Louis, Mississippi, 229 pp.

Wolff G. 1982. A beak key for eight eastern tropical pacific cephalopod species with relationships between their beak dimensions and size. Fishery Bulletin 80(1): 357-370.

Wormuth J. 1976. The biogeography and numerical taxonomy of the oegopsid squid family Ommastrephidae in the Pacific Ocean. Bulletin of the Scripps Institution of Oceanography 23: 1-90.
Wormuth J. 1998. Workshop deliberations on the Ommastrephidae: A brief history of their systematics and a review of the systematics, distribution, and biology of the genera Martialia Rochebrune and Mabille, 1889, Todaropsis Girard, 1890, Dosidicus Steenstrup, 1857, Hyaloteuthis Gray, 1849, and Eucleoteuthis Berry, 1916. In: Voss N, M Vecchione, R Toll \& M Sweeney (eds). Systematic and biogeography of cephalopods. Volumen II. Smithsonian Contribution to Zoology 586(2): 373-383.

Zar J. 1999. Biostatistical analysis, 663 pp. Prentice Hall, Englewood Cliffs.

Recibido el 4 de octubre de 2011 y aceptado el 11 de octubre de 2012

Editor Asociado: Gabriela Muñoz C. 